\title{
La fusion de la neige et les crues de juin 1957 dans les Alpes françaises
}

\section{The melting of snow}

\author{
PAR A. POGGI

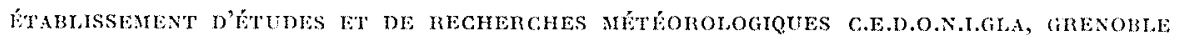

\begin{abstract}
Rappels succincts sur la théorie de la fusion do la neige. Relations permettant de calculer la fusion en fonction de certains parametres ou données physiques.

Application aux crlues de juin 1957 dans les Alpes. Conclusions: Apport moyen de la neige de l'ordre de 40 a $60 \mathrm{mim}$, et maximum certainement inférieur a $80 \mathrm{~mm}$ d'eau de fusion en 24 henres, dont moins d'un alixieme dû aux précipitations liquides, influences de la fusion sur la saturation de la conche nivale et da sol.
\end{abstract}

\begin{abstract}
A brief recapitulation is given of the theory of snowmelt. Expressions are given by means of which snowmelt can be complted in terms of certain factors or physical dafa.

These equations are applied to the June 1957 floods in the Alps. It is shown that mean runoff due to snowmell is of the order of 40 to $60 \mathrm{~mm}$ and that the maximum for 24 hours is certainly less than $80 \mathrm{~mm}$, less than ten percent being atributable to rainfall and to the effects of melting on the saturation of the snow cover and the ground.
\end{abstract}

\section{I. - INTRODUCTION}

En juin 1957, des crues d'une extrême violence provoquèrent de graves dégâts dans les Alpes orientales françaises.

Ce phénomène a donné lieu à diverses études dont l'une par M. Tricart, publiée dans la Revue de Géographie Alpine [1], est consacrée surtout à la crue du Guil; une autre, très intéressante, a été élaborée par les Services Hydrologiques d'E.D.F. [2]. S'il est facile de constater que ces crues se sont déclenchées à la suite de précipitations importantes, on ne peut nier la réalité d'un apport nival. Une fois de plus, s'est posé le problème de l'estimation de cet apport.

M. Tricart nous a surpris par l'énormité des ordres de grandeurs proposés (200 à $300 \mathrm{~mm}$ en $24 \mathrm{~h}$ pour la crue du Guil). L'étude publiée par le C.H.M. «Alpes» d'E.D.F. nous propose, par contre, pour les crues de la haute Iscre et de l'Arc supérieur, des valeurs de 50 à $60 \mathrm{~mm}$, soit environ 20 a $35 \%$ de l'apport total.

M. Pardé aboutit, par des raisonnements fon- dés sur le volume des précipitations, les coefficients d'écoulements probables, selon ses connaissances, après consultation d'un spécialiste, M. Kasser, et discussion avec nous-mêmes, à des pourcentages probables assez analogues.

Nous-mêmes avions, avant tout entretien avec M. Pardé, évalué la fusion probable de la neige par des méthodes purement fondées sur la physique et sur certaines équations assez satisfaisantes, semble-t-il, équations établies expérimentalement par divers chercheurs. On verra que nous avons trouvé des valeurs très proches de celles auxquelles aboutit M. Pardé [3]. Bien entendu, en un tel domaine, vu la rareté relative des bases expérimentales et la non connaissance directe des températures (évaluées par extrapolation), nos valeurs proposées ne peuvent prétendre à mieux qu'à des approximations assez grossières. En pleine connaissance de nos incertitudes, nous avons tenu à présenter les valeurs extrêmes qui nous semblent admissibles pour la fusion et son rôle durant la crue. 


\section{II. - EQUATIONS UTILISARLES POUR LE CALCUL DES FUSIONS NIVALES}

Nous avons utilisé en concurrence diverses équations dont il parait difficile de contester la valeux au moins approximative.

\section{A) Relation proposée par ZingG [4]:}

$$
\mathrm{F}=4,5 \mathrm{~T}
$$

F représente la fusion exprimée en $\mathrm{mm}$ d'eau,

'T représente, soit la température moyenne quotidienne positive pour une période de $24 \mathrm{~h}$, soit le nombre de degrés/jours positifs pour une période de plusieurs jours.

B) Relation proposée par Weiss et WILSON [5]:

$$
\mathrm{F}=5,5 \mathrm{~T}
$$

F et 'T ont la même signification que dans l'équalion précédente

C) Relation tirée de «SNow-Hydrology $》[6]$ :

$$
\mathrm{F}=2,74 \mathrm{~T}+12,2
$$

F représente la fusion en 24 h exprimée en $\mathrm{mm}$ d'eau,

T la température moyenne quotidienne en degrés $C$.

Cette relation est valable pour des températures comprises entre $0,5^{\circ}$ et $20^{\circ}$.

D) Autre RElation Extratte DE «SNOWHYDROLOGY »:

$$
\mathrm{F}=1,83 \mathrm{~T}_{\mathrm{M}}+5,1
$$

$\mathrm{F}$ représente la fusion en $24 \mathrm{~h}$, exprimée en $\mathrm{mm}$ d'eau
' $\mathrm{T}_{\mathrm{ar}}$ est la température maximum en degrés C relevée au cours des $24 \mathrm{~h}$.

Cette relation est valable pour des températures maximums comprises entre $7^{\circ}$ et $25^{\circ}$.

Il existe un certain nombre de relations plus ou moins complexes faisant intervenir différents facteurs, tels que le vent, la pression atmosphérique, le gradient de température au-dessus de la surface de la neige, etc. Nous prions les lecteurs intéressés par ces questions de consulter «Snow-Hydrology».

La figure 1 permet de comparer les trois premières relations. On constate que pour des températures moyennes, comprises entre $4^{\circ}$ et $15^{\circ}$, les valeurs de $\mathrm{F}$ fournies par les trois relations restent comprises dans des limites acceptables.

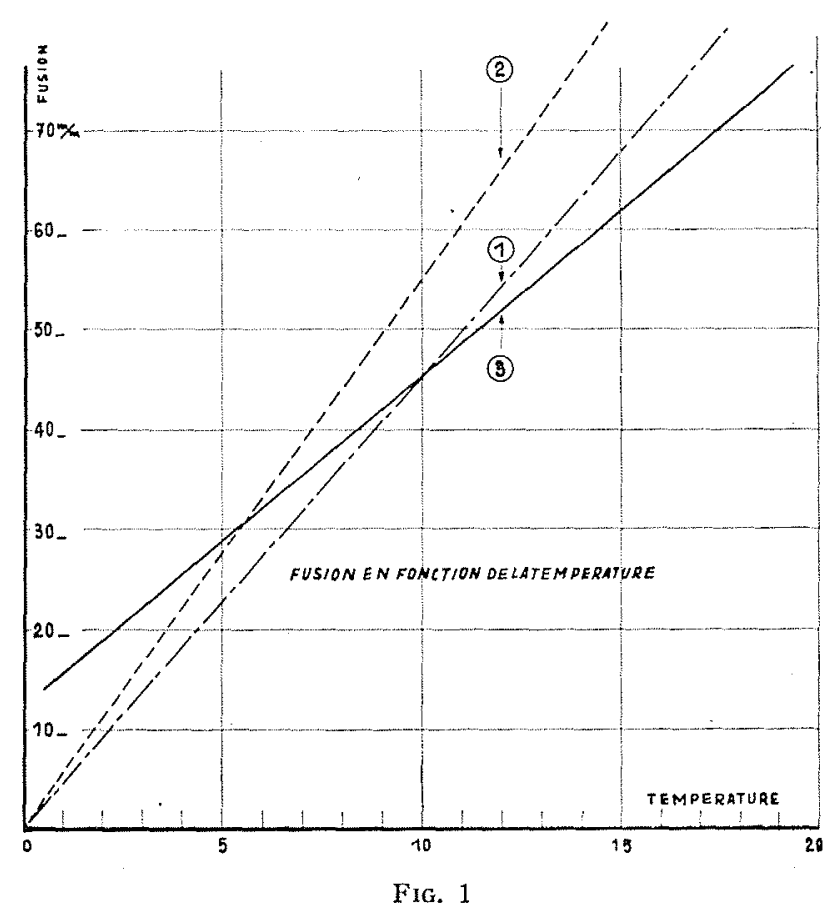

FIG. 1

\section{III. - APPLICATIONS A LA CRUE DE JUIN 1957}

Nous allons, à l'aide des relations précédentes, évaluer en eau, les quantités de neige qui ont pu être liquifiées dans le haut Queyras, la haute Mamrienne et la haute Tarentaise lors de la crue de juin, et même chaque jour du $1^{\text {er }}$ au 15 juin.

\section{A) La CRUe du Guil:}

Nous disposons des observations quotidiennes d'une dizaine de stations.

La seule variable connue ou susceptible d'être 
calculée étant la température, nous n'avons utilisé que les relations où ce paramètre intervient seul :

Relations de Zings:

(1) $\mathrm{F}=4,5^{\mathrm{r}} \mathrm{I}$

Relation tirée de «Snow-Hydrology»:

$$
\begin{array}{ll}
\text { (2) } \mathrm{F}=2,7 \mathrm{~T}+12,2 & 0,5 \leq \mathrm{T} \leq 20,0 \\
\text { (3) } \mathrm{F}=1,8 \mathrm{~T}_{\mathrm{M}}+5,1 & 7^{\circ} \leq \mathrm{T}_{\mathrm{M}} \leq 25^{\circ}
\end{array}
$$

Il faut remarquer que les relations (2) et (3) ne sont valables que pour des espaces complètement déboisés.

Nous prendrons comme bases les valeurs suivantes, évidemment dues à des approximations, qui majorent systématiquement ces valeurs.

a) Températures à $2500 \mathrm{~m}$. - Les températures quotidiennes moyennes et les températures maximums à $2500 \mathrm{~m}$ du $1^{\text {er }}$ au 15 juin, ont été calculées à partir des températures relevées sous abri dans les stations sélectionnées, en adoptant une loi linéaire de décroissance en fonction de l'attitude $(\Delta \mathrm{T}=-0,5$ degré par $100 \mathrm{~m}$ ).

Nous avons, également, pour chaque jour de cette période, déterminé l'ordre de grandeur de l'altitude de l'isotherme $0^{\circ}$, à l'aide des cartes hebdomadaires publiées par le C.H.M. A partir

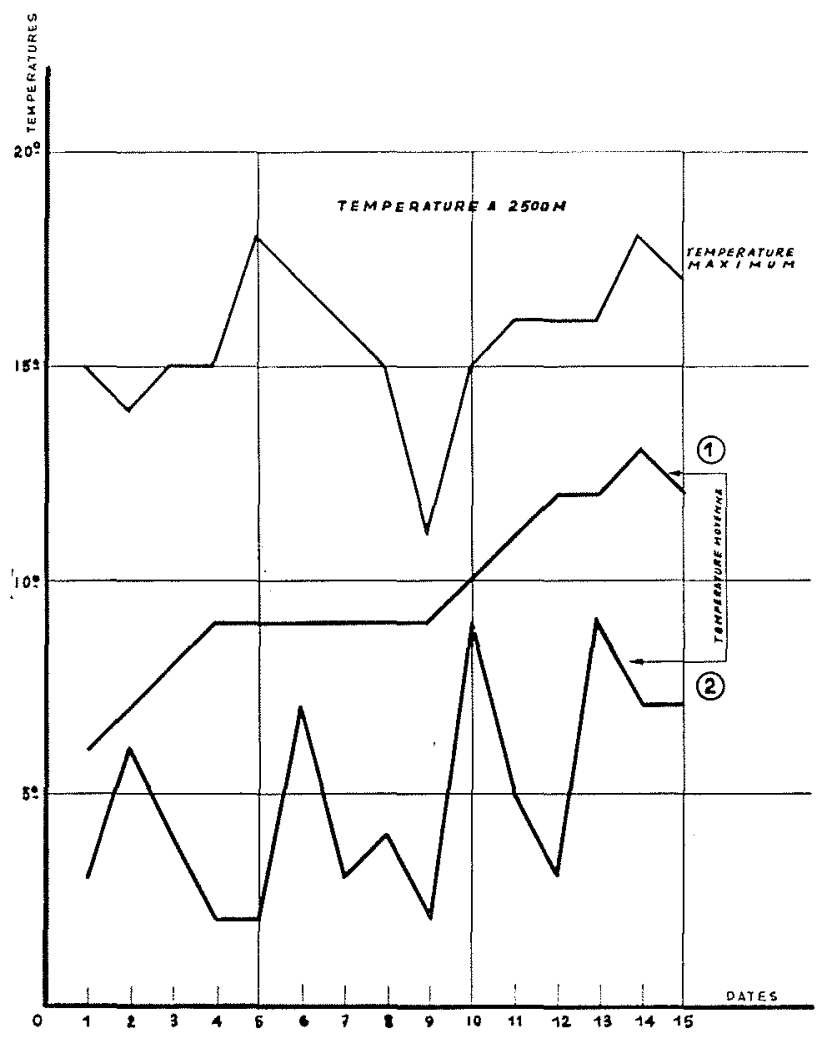

FIG. 2 de cette altitude, en admettant le même gradient de température, nous avons calculé la température à $2500 \mathrm{~m}$.

Nous disposons alors quotidiennement de 10 températures; nous avons choisi arbitrairement, comme valeur représentative de la température quotidienne sur l'ensemble du bassin, la plus grande de ces 10 valeurs (fig 2 ).

Nous avons calculé, à partir de la température et de l'altitude du niveau $700 \mathrm{mb}$, des températures moyennes plus proches sans doute de la réalité. Evidemment, il nous est difficile de tenir compte des inversions possibles de températures. $\left({ }^{*}\right)$

Sur la figure 2 nous avons représenté les courbes de températures déterminées à l'aide de deux méthodes et la courbe des températures maximums. On constate que la courbe (1) établie à l'aide de la première méthode est nettement au-dessus de celle (2) qui a pu ètre tracée a l'aide des résultats fournis par la seconde.

b) Calcul de la fusion due aux seuls échanges de chaleur entre la neige et l'air. - L'application des relations (1), (2) et (3) fournit les valeurs de la fusion en $24 \mathrm{~h}$. Voir figure 3 .

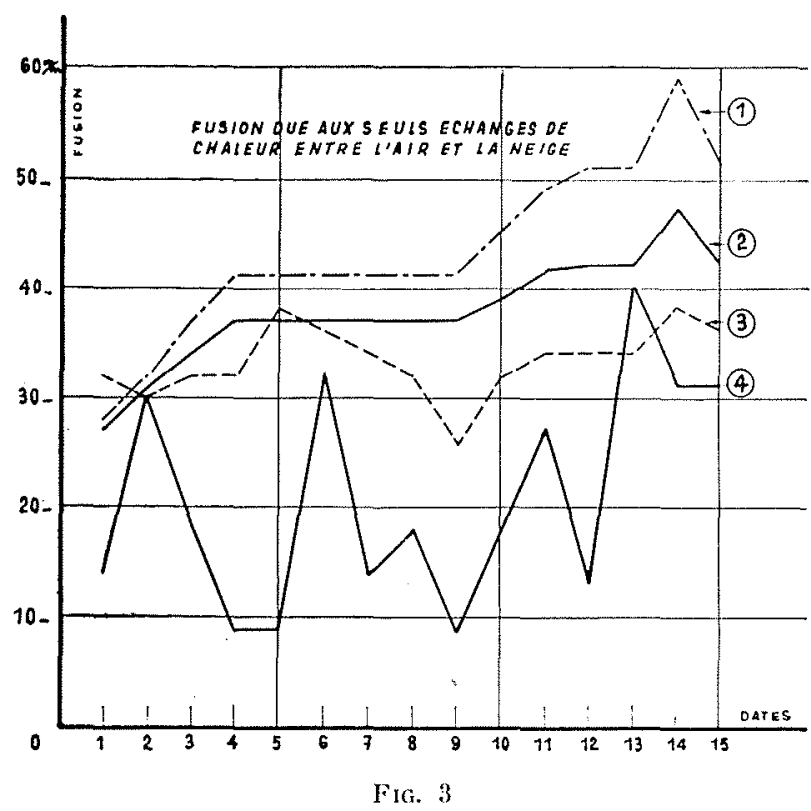

L'application de la relation de Zingg aux températures calculées à l'aide de la deuxième méthode permet d'établir la courbe (4) de la figure 3 .

c) Examen des résultats. - Nous constatons que, pendant la $1^{\text {re }}$ décade, la fusion a été in-

(*) Nous avons utilisé les radiosondages effectuées i Lyon-Payerne et Milan. 
lense et que par conséquence il n'y a pas eu conservation de la neige. Il est probable qu'au cours de cette période, l'évaporation n'a pas été négligeable, limitant la fusion mais provoquant tout de même la diminution du stock neigeux.

Si nous acceptons l'hypothèse d'une valeur en cau du stock neigeux de 300 à $400 \mathrm{~mm}$ entre $2000 \mathrm{~m}$ et $2500 \mathrm{~m}$ au $1^{\text {er }}$ juin, et si nous calculons les quantités cumulées d'eau de fusion du $1^{\text {er }}$ au 15 juin, dans les conditions admises ci-dessus, nous trouvons :

$644 \mathrm{~mm}$ d'après la courbe (1) $566 \mathrm{~mm}$ d'après la courbe (2) $500 \mathrm{~mm}$ d'après la courbe (3) $332 \mathrm{~mm}$ d'après la courbe (4)

Aussi, sous l'hypothèse admise plus haut nous voyons que les quantités d'eau de fusion données par les courbes (1), (2) et (3) sont nettement surestimées, et que par contre la quantité cumulée de fusion d'eau calculée à l'aide de la courbe (4) est bien comprise entre les limites admises.

Quoi qu'il en soit, puisque notre but est de fixer une limite supérieure du volume quotidien d'eau de fusion, nous pouvons admettre que celui-ci est donné par la courbe (1) de la figure 3 .

L'examen'du graphique 'montre que le maximum de la fusion s'est produit le 14 , et que le volume est de l'ordre de $60 \mathrm{~mm}$; mais il s'agit la d'un maximum, le volume plus probable étant de l'ordre de $40 \mathrm{~mm}$ en $24 \mathrm{~h}$.

d) Fusion due à la chaleur cédée par les précipitations. - Etant donné qu'il est pratiquement impossible de connaître les quantités de pluie tombées à $2500 \mathrm{~m}$, nous avons retenu comme valeur des précipitations sur l'ensemble du bassin, celles qui ont été recueillies dans les stations les plus arrosées (fig 4).

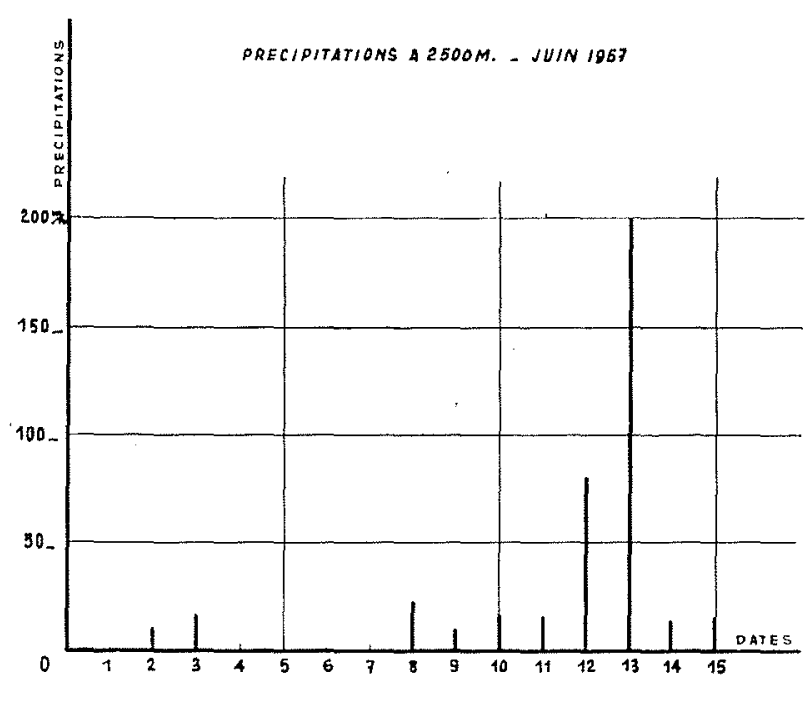

FIG. 4

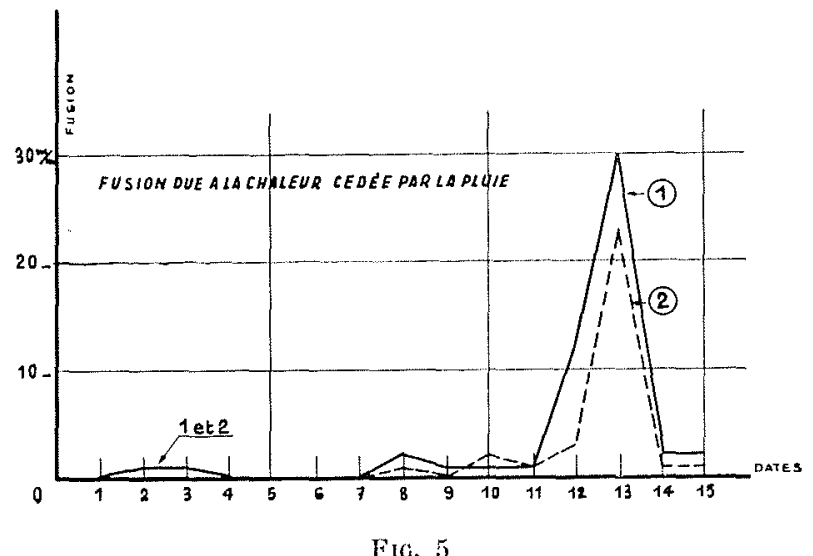

Connaissant le volume de la pluie et sa température, il est facile de calculer la chaleur recue par la neige (nous admettrons que la neige est $\dot{a} 0^{\circ}$ ) et par conséquent la quantité d'eau de fusion.

Nous donnons, figure 5 , les valeurs des lames d'eau de fusion qui sont nettement surestimées en (1), car calculées à l'aide des températures déterminées par la 1 re méthode, et en (2) les valeur's des lames d'eau calculées à l'aide des températures déterminées par la $2^{\circ}$ méthode. Pour rendre plus facile la lecture du diagramme nous avons relié les points par des segments de droite.

Il convient de remarquer que la fusion provoquée par la chaleur cédée par les précipitations est très faible. On constate aisément que la quantité cumulée de l'eau de fusion est inférieure à $55 \mathrm{~mm}$ (courbe 1 , figure 5 ) soit environ $10 \%$ de la fusion due aux seuls échanges de chaleur entre l'air et la neige (500 à $644 \mathrm{~mm})$.

D'après la courbe (2), figure 5, la quantité cumulée d'eau de fusion due à la pluie est inférieure à $35 \mathrm{~mm}$, soit encore $10 \%$ de la fusion dues aux échanges de chaleur entre l'air et la neige : $332 \mathrm{~mm}$. Il est en outre certain que l'effet mécanique de la pluie tend à accroître la fusion, mais, d'une façon générale, cette action doit être de peu d'importance. Quoi qu'il en soit, il nous est impossible d'en fixer une valeur.

e) Lame de fusion totale. - La figure 6 donne la fusion totale calculée d'une part à l'aide des courbes (1) figure 3 et (1) figure 5 , et d'autre part des courbes (4) figure 3 et (2) figure 5.

L'examen de la courbe (1) de la figure 6 montre que durant la $1^{\text {re }}$ décade la fusion est de l'ordre de 30 à $40 \mathrm{~mm}$ en $24 \mathrm{~h}$, de l'ordre de 40 à $60 \mathrm{~mm}$ au cours des jours suivants, avec un maximum de l'ordre de $80 \mathrm{~mm}$ le 13 . Etant donné l'ordre de grandeur des températures admises à $2500 \mathrm{~m}$, il s'agit fort probablement d'une quantité majorant la fusion réelle. La courbe (2) de la figure 6 donne certainement 


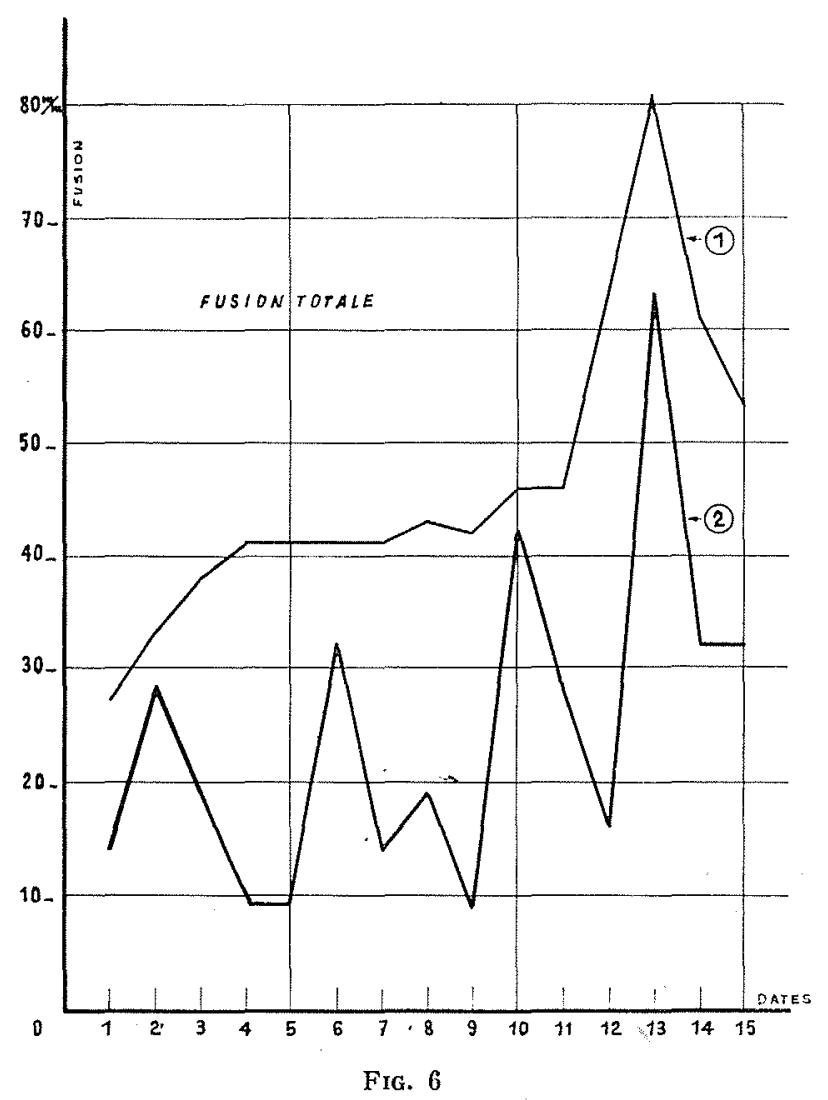

des valeurs beaucoup plus probables de la fusion. On constate qu'au cours de la première décade, les valeurs de la fusion oscillent entre 10 et $30 \mathrm{~mm}$ en $24 \mathrm{~h}$; au cours des jours suivants, les fluctuations sont plus accentuées, et on note un maximum, le 13 , qui atteint $60 \mathrm{~mm}$ en $24 \mathrm{~h}$.

Nous retrouvons là l'ordre de grandeur donné par le C.H.M. pour la crue de la haute Isère et de l'Arc supérieur en juin 1957.

B) Les CRues de la haUte Isère, et de l'Arc SUPÉRIEUR :

Nous avons, en utilisant une méthode analogue à la précédente, calculé les températures à $2500 \mathrm{~m}$ pour les bassins de l'Arc supérieur et de la haute Isère.

La valeur des précipitations quotidiennes, à $2500 \mathrm{~m}$, a été arbitrairement estimée à une quantité de $50 \%$ supérieure à la plus forte précipitation quotidienne recueillie dans la station la plus arrosée. La fusion due aux précipitations étant infime, nous n'avons calculé cette fonte que pour les précipitations des 12, 13, 14 et 15 juin. Nous admettrons également que l'ordre de grandeur de cette fusion (due aux pluies) est le même en Maurienne qu'en Tarentaise. Nous avons, en outre, utilisé une seule relation donnant la fusion en fonction de la température, celle de Zingg.
En application de ces données, on constate qu'au cours de la première décade de juin, la fusion est forte et du même ordre de grandeur que lors des jours suivants. La fusion au cours de la crue n'a rien d'exceptionnel, tout au moins en Maurienne; en Tarentaise, il semble que la fusion ait été un peu plus abondante au cours de la deuxième décade (fig. 7).

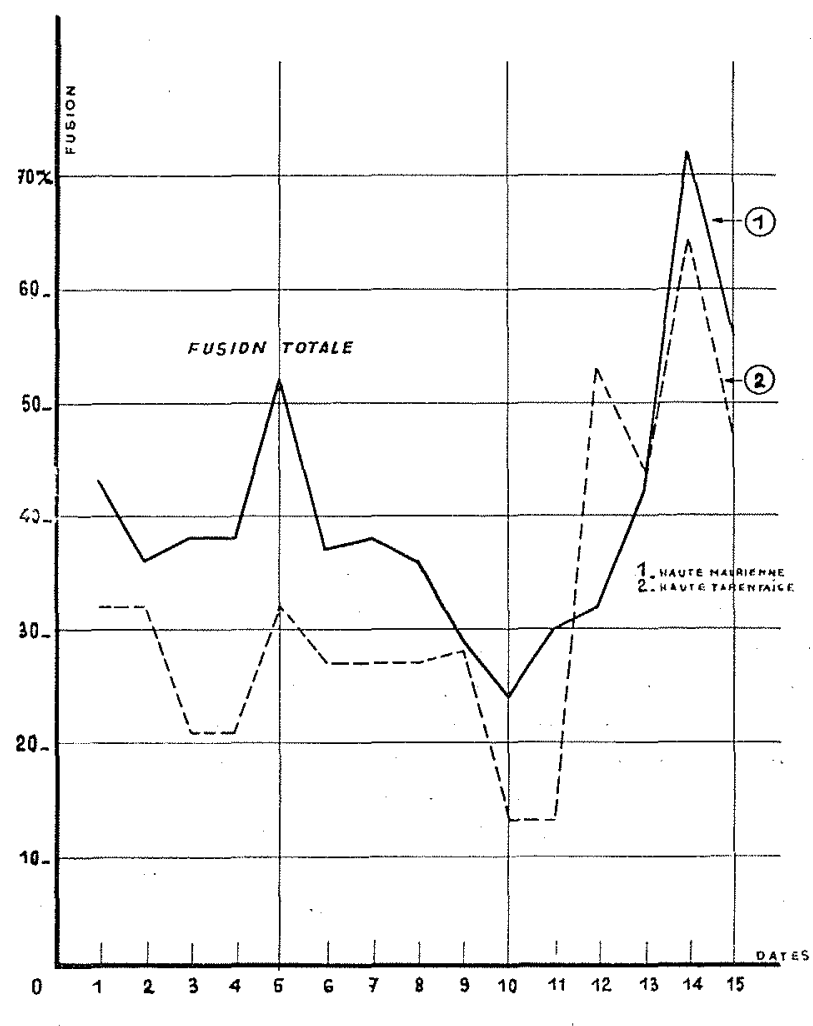

FIG. 7

Nous n'avons pas tenu compte de l'évaporation qui a dû se produire au cours de la première décade. Ce phénomène a pu, selon son intensité, ralentir la fusion, sans que pour autant il y ait eu conservation du stock neigeux; il n'a pu que réduire encore la part de la neige dans la crue. Mais, quoi 'qu'il en soit, il n'y a eu ni conservation du stock au cours de la première décade, ni fusion anormale au cours de la seconde.

Le maximum de fusion a dû survenir le 14 juin, aussi bien en Maurienne ( $72 \mathrm{~mm}$ en $24 \mathrm{~h}$ ) qu'en Tarentaise $(64 \mathrm{~mm}$ en $24 \mathrm{~h})$. Nous répèterons, une fois de plus, qu'il s'agit là de quantités majorant très certainement la fusion réelle. Nous retrouvons l'ordre de grandeur de la fusion maxima calculée par le C.H.M.

Isère à Tignes......... $50 \mathrm{~mm}$ Isère au Pont de Laisinant.. $60 \mathrm{~mm}$ Bissorte. . . . . . . . . $60 \mathrm{~mm}$ 


\section{V. - CONCLUSIONS}

Dans tous nos calculs, nous avons surestimé les valeurs des températures à $2500 \mathrm{~m}$, et par conséquent, les valeurs des lames d'eau de fusion ont été majorés.

En ce qui concerne le Guil, nous trouvons que la valeur totale de la lame d'eau de fusion du $1^{\mathrm{er}}$ au 15 juin varie, en fonction des relations utilisées, de $550 \mathrm{~mm}$ à $700 \mathrm{~mm}$. Or, si l'évaluation du stock neigeux au $1^{\text {er }}$ juin (300 à $400 \mathrm{~mm}$ entre 2200 et $2500 \mathrm{~m}$ ) est exacte, nos propres estimations sont nettement exagérées. Si, par contre, nous examinons les résultats calculés à l'aide de la courbe (2) figure 5 (lame de fusion calculée à partir des températures mesurées par radio-sondages et réduites à $2500 \mathrm{~m}$ ), nous constatons que la lame d'eau de fusion correspond à $332 \mathrm{~mm}+33 \mathrm{~mm}=365 \mathrm{~mm}$. Cette quantité est du même ordre de grandeur que l'estimation du stock neigeux: Il nous parait donc possible d'affirmer que les valeurs suivantes :

40 à $50 \mathrm{~mm}$ du 10 au 11 et du 11 au 12 juin, 50 à $60 \mathrm{~mm}$ du 12 au 13 ,

70 à $80 \mathrm{~mm}$ du 13 au 14 ,

50 à $60 \mathrm{~mm}$ du 14 au 15 ,

sont des bornes supérieures difficilement admissibles et que, par contre, l'ordre de grandeur des lames d'eau de fusion probable est le suivant:

\section{$40 \mathrm{~mm}$ du 10 au 11 juin, $30 \mathrm{~mm}$ du 11 au 12 , \\ 15 à $20 \mathrm{~mm}$ du 12 au 13 , \\ $60 \mathrm{~mm}$ du 13 au 14 , \\ $30 \mathrm{~mm}$ du 14 au 15.}

Du 12 au 15 juin nous pouvons donc admettre comme probable une lame de fusion de $110 \mathrm{~mm}$, et comme un maximum difficilement atteint la quantité suivante : $170 \mathrm{~mm}$ à $200 \mathrm{~mm}$. La valeur moyenne probable pour la période du 12 au 15 juin est de l'ordre de :

$$
\frac{110 \mathrm{~mm}}{3} \simeq 40 \mathrm{~mm} \text { par jour. }
$$

Nous allons comparer nos résultats avec ceux de M. Tricart.

Cet auteur estime de 200 à $300 \mathrm{~mm}\left(^{\star}\right)$ la quantité de neige fondue le 13 , et de 100 à $120 \mathrm{~mm}$ la quantité fondue le 14. L'ordre de grandeur pour la lame d'eau de fusion en $48 \mathrm{~h}$ serait donc compris entre 300 et $400 \mathrm{~mm}$, ce

(*) D'après la dernière ligne de la page 608 dans larticle de M. Tricart. Et, à la page 609 , nous lisons : « Dans les hautes vallées, proches de la frontière, ce sont donc des tranches d'eau de 200 a $400 \mathrm{~mm}$ qui ont èté libérées. 》 qui dor erait en $24 \mathrm{~h}$ à peu près 150 à $200 \mathrm{~mm}$. La plus petite de ces deux valeurs est presque 4 fois supérieure à la quantité que nous avons admise. Si en outre nous calculons la lame maximum (sclon nos estimations) d'eau de fusion totale pour les 13 et 14 juin, nous trouvons $81+61=142 \mathrm{~mm}$, soit $70 \mathrm{~mm}$ en $24 \mathrm{~h}$, c'est-àdire moins que la moitié de la valeur minimum proposée par M. Tricart.

En ce qui concerne la haute Maurienne et la Tarentaise, nous avons trouvé des maxima de l'ordre de $72 \mathrm{~mm}$ en $24 \mathrm{~h}$ et de $64 \mathrm{~mm}$ en $24 \mathrm{~h}$, soit un peu moins que pour le Guil.

Des quantités supérieures à celles qui ont été calculées paraissent inacceptables. Nous estimons comme probables, au cours des périodes où la fusion a été la plus intense, des lames d'eau de $60 \mathrm{~mm}$ en $24 \mathrm{~h}$ et comme borne supérieure, des lames d'eau de $80 \mathrm{~mm}$ en $24 \mathrm{~h}$.

Remaroue. -- Il ressort du tableau publié page 243 de «Snow-Hydrology» que les fusions maximums observées sur une série de plusieurs années sont les suivantes:

\begin{tabular}{|c|c|}
\hline Labonatorus: & $\begin{array}{c}\text { Fusion maxima } \\
\text { pour 1 degré/jour } \\
\text { positif en prenant } \\
\text { comme base } \\
\text { la temperature } \\
\text { moyenne }\end{array}$ \\
\hline Central Sierra Snow Laboratory... & $5,5 \mathrm{~mm}$ \\
Upper Columbia Snow Laboratory.. & $6,6 \mathrm{~mm}$ \\
Willamette Basin Snow Laboratory. & $5,5 \mathrm{~mm}$ \\
\hline
\end{tabular}

Si nous calculons la quantité maximum d'eau de fusion qui a pu s'écouler les 13 et 14 juin en fonction, d'une part, de la plus forte de ces trois valeurs, à savoir $6,6 \mathrm{~mm}$, et des plus fortes températures moyennes à $2500 \mathrm{~m}$, nous trouvons :

$$
\begin{aligned}
& \text { le } 13 \text { juin } F=6,6 \times 12=79,1 \mathrm{~mm} \\
& \text { le } 14 \text { juin } \mathrm{F}=6,6 \times 13=85,8 \mathrm{~mm}
\end{aligned}
$$

Si maintenant nous utilisons les températures moyennes à 2500 déterminées par la seconde méthode, nous avons :

$$
\begin{aligned}
& \text { le } 13 \text { juin } \mathrm{F}=6,6 \times 9=59,4 \mathrm{~mm} \\
& \text { le } 14 \text { juin } \mathrm{F}=6,6 \times 7=46,2 \mathrm{~mm}
\end{aligned}
$$

Le tableau suivant extrait de la page 244 de l'ouvrage cité nous donne la fusion maximum 
observée pour 1 degré/jour en prenant comme base de la température maximale.

\begin{tabular}{|c|c|}
\hline & $\begin{array}{c}\text { Fusion maxima } \\
\text { Laboratorke } \\
\text { pour } 1 \text { degré/jour positif } \\
\text { en prenant comme base } \\
\text { la température maximale }\end{array}$ \\
\hline C. S. S. L........ & $2,7 \mathrm{~mm}$ \\
U. C. S. L........ & $2,0 \mathrm{~mm}$ \\
W. B. S. L........ & $3,0 \mathrm{~mm}$ \\
\hline
\end{tabular}

Calculons en fonction de la plus grande de ces trois valeurs, d'une part, et la température maximum, d'autre part, la fusion maximum des 13 et 14 juin :

$$
\begin{aligned}
& \text { le } 13 \text { juin } \ldots \quad F=3,0 \times 16=48 \mathrm{~mm} \\
& \text { le } 14 \text { juin } \ldots . \quad F=3,0 \times 18=54 \mathrm{~mm}
\end{aligned}
$$

Ces résultats, dans la mesure où les valeurs admises sont applicables, confirment bien nos conclusions.

\section{BIBLIOGRAPHIE}

[1] Tricart (J.). - La crue de la mi-juin 1957 sur le Guil, l'Ubaye et la Cerveyrette (R.G.A., tome XLVI, 1958 , fase. 4 ).

[2] E.D.F. - C.H.M. " Alpes ». - Etude de la crue de juin 1957.

[3] PandF́ (M.). - Sur les fontes des neiges lors des crues. La grande crue alpestre de juin 1957. Quelques données. - R.G.A.

[4] ZingG (F.). - Beitrag zur Kenntnis des Schmelzwasserabflusses der Schneedecke.
[5] Weiss (Léonard L.) et WiLson (Walter T.). - Snowmelt degree-day ratios determined from snow-lab date. (Transactions American Geophysical Union, vol. 39 , fasc. 4 , août 1958 .)

[6] Snow Hydrology. - North Pacific Division, Corps of Engineers U.S. Army, chap. 5 et 6.

[7] Wagner (Jean). - La crue du printemps dans le bassin de la haute Durance (fasc. dactylographié).

\section{DISCUSSION}

Président: M. DurfauT

M. le Président remercie M. Porar et oure la diseussion.

M. Bannillon cite une phrase de l'Annuaire Hydrologique de la France 1957 , relative à la région de l'Ubaye :

«Bien que cette crue intervienne en période de fusion, il ne faut pas exagérer la part de la neige, qui ne semble pas avoí foumi plus du tiers du débit sur le Guil et du quart sur l'Isère et l'Arc supérieur. »

M. BARRILlon demande à M. Pogar de bien vouloir cxprimer la proportion de la neige fondue par rapport aux débits de crue dans des termes parallèles à ceux de l'Annuaire; on pourrait dire, par exemple, que la fusion de la neige ne semble pas avoir fourni plus de $1 / \boldsymbol{x}$ pour le débit du Guil et $1 / y$ pour le débit de l'Isère et de l'Arc, et indiquer les valeur's de $x$ et $y$.

M. Pardé se déclare d'accord avec les ordres de grandeur avancés pour la part de la fonte des neiges lor's de la crue éprouvée par le Guil du 12 au 14 juin 1957. Une lettre du spécialiste suisse réputé M. Kasser, indique la vraisemblance de quantités analogues.

Plus amples détails seront donnés dans la Revne de Gégraphie Alpine où vont paraître l'article de M. Pogar, et un article de M. PArpé lui-même, qui étudie la crue de 1957 d'après les débits tels qu'on peut les évaluer assez grossièrement.

On peut admettre, pour le débit de fonte maximum du Guil, $1000 \mathrm{~m}^{3}$, encore qu'un ingénieur très expert pour l'évaluation des débits, M. André, juge improbable plus de $700 \mathrm{~m}^{3}$ à la Maison du Roi. Le total des débits moyens journaliers en trois ou quatre jours a pu atteindre 1100 à $1200 \mathrm{~m}^{3}$ au plus. Le chiffre de $3000 \mathrm{~m}^{3}$
(3 fois $1000 \mathrm{~m}^{2}$ ) en trois jours $\mathrm{n}^{3} \mathrm{a}$ pas le plus mince fondement de vaaisemblance à moins que l'Electricité de France ait sous-estimé de $50 \dot{a} 60 \%$, sinon plus, les débits totaux colrespondants de la Durance a Ventavon, l'Archidiacre et Serre-Poncon pour un bassin cing fois supérieur à celui du Guil.

M. PaRnE admet comme valable, en gros, le chiffre donné pour Serre-Poncon, soit $2200 \mathrm{~m}^{\mathrm{s}}$ en trois jours. Ce total dépasse celui auquel on arrive aux deux autres stations voisines.

Après divers calculs, M. Parbé juge acceptable, en admettant $60 \%$ pour l'écoulement de la pluie liquide, une proportion de 28 à $37 \%$ pour la neige fondue par rapport au volume liquide total de la crue du Guil en quatre jours. L'apport nival representerait encore, point trop exactement, 25 a $30 \%$ du plus fort volume liquide en 24 heures, et 20 à $25 \%$ au plus du débit de pointe. Tapportée à la surface supposée enneigée, soit à celle qui dépasse 2250 mètres d'altitude, la neige semble avoir pu fournir 60 à 70 millimètres lors des 24 heures de fusion la plus intense, renforcée par l'effet de la pluie; et $90 \mathrm{~mm}$ si le coefficient d'écoulement de la pluie seule a été de $50 \%$, chiffre qui, d'après diverses comparaisons, paraît trop faible. Or, certains ont prétendu que la fonte avait pu fournir en un jour 200 à 300 millimetres on 300 et 400 a 520 millimetres en trois jours.

M. PARDÉ a cherché aussi à évaluer la part de la fonte lors de grosses crues arrivées depuis 40 ans sur l'Isère avant Grenoble, la Valloirette, le Rhône alpestre, la Massa, et la Visp, par le seul fait de chaleurs caniculaires, c'est-à-dire très supérieures aux températures qui 
ont agi en juin 1957. Il trouve que les surfaces enneigées, et englacées au-dessus de 2250 ou 2500 mètres selon les cas, ont pu fournir, sur les bassins les plus exposés au soleil, en un jour, 65 à 70 millimètres d'eau de fusion (50 à 55 au plus sur la Visp exposée au nord) avec des maxima locaux de l'ordre de 90 , peut-être, sur certains versants face au sud. Il semble que les erreurs it eraindre dans ces évaluations ne puissent représenter un pourcentage très considérable.

Enfin, M. PAndÉ a cherché quelques hypothèses pour les fontes de neige qui alimentent certaines crues canadiennes ou des Etats-Unis et d'autres sur les crues soviétiques.

M. Barrillon pense qu'il serait intéressant de voir le calcul de M. Kasser, et de savoir la proportion de surface enneigée dont il s'est servi.

M. PARDÉ précise que M. Kasser a simplement indiqué que vers $2500 \mathrm{~m}$, la quantité de neige qui peut avoir fondu en 24 heures de juin 1957 , est de 60 ou $70 \mathrm{~mm}$ d'eau au grand maximum à une altitude correspondant à la moyenne de la surface enneigẻe.

M. Moriat signale, pour répondre à la question initiale de M. Barrillon, qu'il semble y avoir excellent accord entre les conclusions de M. Pogar et les affirmations de l'Annuaire Hydrologique : le rôle de la fusion de la neige dans l'écoulement de la crue de juin 1957 est bien du même ordre de grandeur.

M. Pogar précise qu'il s'est intéressé seulement au cralcul de la quantité de neige fondue, sans chercher à connaître les débits.
M. Pardé tient à faire remarquer que, dans son étude qui est bien plus un essai, il n'a pas cherché à présenter sur les neiges fondues lors de diverses intumescences fluviales, des chiffres auxquels il attribuerait une exactitude rigoureuse, y compris celle qui concemerait plusieurs décimales. La fausse précision sur des phénomènes si difficiles à saisir ou à exprimer par des formules impeccables ne l'a jamais séduit, ni impressionné et quand lui-même donne des chiffres très précis, e'est sans illusion. Il a seulement voulu chercher des ordres de grandeur pour les lames d'eau dues à la fonte en diverses circonstances. Le chiffre maximum à admettre pour 24 heures du 13 au 14 juin 1957 lui semble en somme être compris entre 50 et 100 millimètres. En proposant des valeurs aussi écartées, dans une sorte de boutade, M. Pardé estime que la plus énorme de ces quantités serait le tiers de celle qu'avancent certains auteurs. En réalité, il juge vraisemblable qu'il faut retenir all plus 60 à 70 millimètres avec des chances sérieuses d'exagération, pour des cas où l'exactitude parfaite est aussi peu saisissable.

M. Barrilion demande que, dans la publication, l'auteur mette un graphique des trois formules de fusion qu'il a employées afin de voir exactement ce que donne chacune d'elles.

M. Pogri indique quelques exemples extraits de son mémoire et que l'on trouvera dans celui-ci.

Ces chiffres correspondent d'ailleurs à l'ordre de grandeur calcule par E.D.F. pour la crue de l'Arc et de l'Isère.

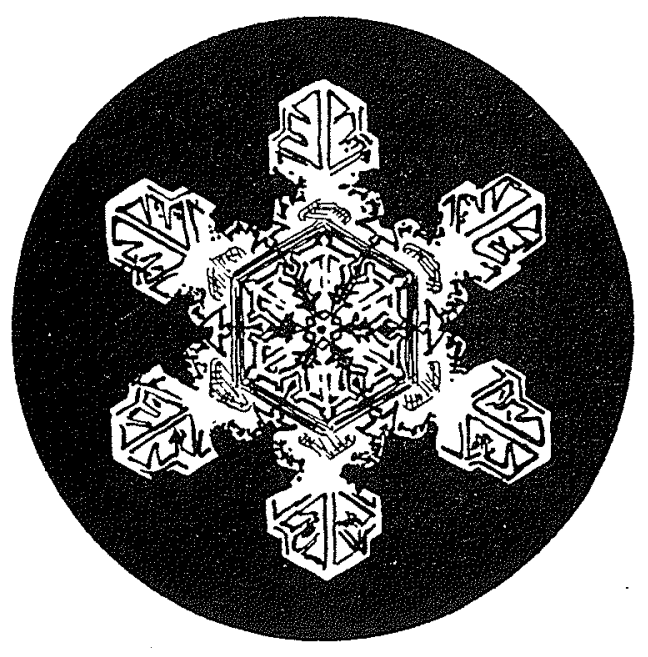

